\title{
L'élection présidentielle de février 2019 au Sénégal
}

Entre renouveau de l'échiquier politique et percée du vote régionaliste

Mamadou Bouna Timera, Momar Diongue et Ousmane Thiam

\section{OpenEdition}

Journals

Édition électronique

URL : http://journals.openedition.org/echogeo/18183

ISSN : 1963-1197

Éditeur

Pôle de recherche pour l'organisation et la diffusion de l'information géographique (CNRS UMR 8586)

Référence électronique

Mamadou Bouna Timera, Momar Diongue et Ousmane Thiam, «L'élection présidentielle de février

2019 au Sénégal », EchoGéo [En ligne], Sur le Vif, mis en ligne le 19 décembre 2019, consulté le 26 décembre 2019. URL : http://journals.openedition.org/echogeo/18183

Ce document a été généré automatiquement le 26 décembre 2019.

\section{(c) $(1) \Theta \Theta$}

EchoGéo est mis à disposition selon les termes de la licence Creative Commons Attribution - Pas d'Utilisation Commerciale - Pas de Modification 4.0 International 


\section{L'élection présidentielle de février 2019 au Sénégal}

Entre renouveau de l'échiquier politique et percée du vote régionaliste Mamadou Bouna Timera, Momar Diongue et Ousmane Thiam

\section{Introduction}

1 Le Sénégal est crédité d'une grande maturité démocratique. Sa stabilité politique et ses élections apaisées en sont l'illustration. Mais la « leçon de Dakar » (Soudan, 2010) a été sérieusement écorchée lors de l'élection présidentielle du 24 février 2019, lorsqu'une succession de conférences de presse, le soir même du scrutin, a créé le sentiment d'une possible crise post-électorale. Dès la publication des premières tendances, les étatsmajors des partis et coalitions politiques ont commencé à s'agiter. À 23h15, l'opposition regroupée autour d'Ousmane Sonko et d'Idrissa Seck a annoncé un deuxième tour et mis en garde le pouvoir et certains groupes de presse à son service contre toute forme de confiscation du pouvoir. A 00h05, c'est autour du Premier ministre, Mahammed Boun Abdallah Dione, d'annoncer la réélection du candidat Macky Sall qu'il crédite d'un minimum de 57 \% des voix. Même si la Cour suprême du Sénégal a plus que confirmé le verdict annoncé par le Premier ministre, directeur de la campagne du candidat Macky Sall, cette situation a pendant presqu'une semaine installé le pays dans une tension électorale relayée par des appels à protestation et des déclarations intempestives, au point que le président du Conseil constitutionnel a dû intervenir pour déclarer que son institution est la seule instance compétente pour proclamer les résultats d'une élection.

2 Cette tension n'est pas surprenante. Elle découle d'un contexte préélectoral complexe marqué par plusieurs points de désaccords entre les partis et coalitions de partis, mais aussi de nouveaux rapports de force dans le paysage politique. D'abord, les poursuites judiciaires puis les condamnations de Karim Wade et de Khalifa Sall, de même que la révocation d'Ousmane Sonko de la fonction publique, ont été perçues par certains comme des moyens juridiques et administratifs pour éliminer des adversaires et donc peser sur le jeu électoral. Ensuite, la modification du processus électoral, avec 
l'introduction contestée du parrainage citoyen, a été un facteur de reconfiguration de la carte électorale du Sénégal. Cela a abouti à la diminution drastique du nombre des candidatures. De plus, les femmes ont complètement disparu de la liste officielle des candidats. L'opposition sénégalaise change de stratégie en formant des coalitions dès le premier tour autour d'Idrissa Seck, d'Ousmane Sonko et de Madické Niang. Une autre caractéristique de l'élection présidentielle de 2019 est le retrait des partis classiques. Pour la première fois depuis la fin des années 1970, le Parti socialiste (PS) et le Parti Démocratique sénégalais (PDS) n'ont pas présenté de candidat à une élection présidentielle. Au pouvoir jusqu'en 2000 et principal parti d'opposition jusqu'en 2012, le PS s'est dissous dans la coalition Benno Book Yakaar, dirigée par l'Alliance pour la République (APR), le parti au pouvoir. Quant au PDS, arrivé en rang dispersé à l'épreuve du parrainage, il n'a pu participer à l'élection de 2019 à cause de l'inéligibilité de son candidat, Karim Wade.

3 Si la tension résulte de ce contexte particulièrement bouillonnant, elle peut aussi se comprendre à la lumière des premiers résultats. Il est apparu deux phénomènes qu'il est intéressant d'analyser : la territorialisation des votes, assimilée à tort ou à raison à un vote régional ou ethnique, qui a conforté certains candidats dans leur emprise territoriale et la situation de ballotage dès l'annonce des premiers résultats par la presse, qui aurait pu leur laisser espérer un second tour.

4 L'objectif de l'article est d'analyser le contexte pré-électoral qui permet de comprendre par la suite les positions et appréciations des protagonistes pendant l'élection. Il étudie aussi le processus et les résultats du scrutin avec en retour un regard sur les implications du contexte de déroulement de l'élection. La méthode de recherche associe les démarches quantitative et qualitative. L'analyse est descriptive et s'appuie sur le recueil et la cartographie des données statistiques publiées par le Conseil constitutionnel du Sénégal. Cette approche permet une lecture spatiale du vote à partir du maillage départemental. Le choix du département n'est pas fortuit. Il est le premier échelon administratif de comptage et d'agrégation des résultats après le bureau et le centre de vote. Mais surtout, il renvoie aux territoires d'appartenance socio-ethnique et religieuse; ce qui en fait un indicateur pertinent de vote régionaliste ou ethnique. Cette approche statistique et descriptive est combinée à l'observation et à l'appréciation du contexte électoral, qui permettent de donner sens aux faits généraux et contextuels qui sont à l'œuvre dans la configuration spatiale du vote.

\section{Un contexte envenimé par la crise politico-judiciaire et une organisation chaotique des législatives de 2017}

5 La présidentielle de 2019 s'est déroulée dans un contexte politique envenimé par les dysfonctionnements constatés lors des législatives de 2017, notamment la conduite des opérations pré-électorales (révision des listes, distribution des cartes d'électeurs, parrainage citoyen, etc.) et les condamnations de Karim Wade et de Khalifa Sall.

\section{Une crise politico-judiciaire responsable de l'inéligibilité de Karim Wade et Khalifa Sall}

6 Karim Meïssa Wade, ancien ministre d'État, est le symbole de la politique de lutte contre l'enrichissement illicite du président Macky Sall, élu en 2012. Connu sous le 
sobriquet moqueur de "ministre du ciel, de la terre et de la mer", en raison des responsabilités démesurées que lui avait confiées son père, Abdoulaye Wade, en tant que président de la République, Karim Wade est incarcéré le 17 avril 2013. La cour de répression de l'enrichissement illicite (CREI) l'a condamné, le 23 mars 2015, à six ans de prison ferme, à la saisie de ses biens supposés acquis illégalement et à une amende de 210 millions d'euros. Mais, en juin 2016, Karim Wade est gracié ; depuis, il vit en exil au Qatar, ce qui donne l'impression d'avoir été la condition sine qua non à sa grâce.

7 Les nombreux rebondissements de cette affaire judiciaire, par exemple le refus en septembre 2016 du tribunal correctionnel de Paris d'autoriser la confiscation de biens de Karim Wade ${ }^{1}$, formulée par la justice sénégalaise, confortent la thèse du complot politique brandie par le PDS. Ce feuilleton judiciaire d'une durée de plus de deux ans n'a fait qu'accentuer la popularité de Karim Wade auprès de l'opinion nationale, en l'absence de preuves tangibles des détournements qui lui sont reprochés.

8 Après l'exil de Karim Wade au Qatar, l'affaire Khalifa Sall, maire de Dakar, a pris le relais. Le rapport d'audit de l'Inspection Générale d'État (IGE) a révélé l'utilisation a posteriori de factures douteuses servant à justifier l'utilisation de la caisse d'avance de la ville de Dakar, une enveloppe financière mensuelle de 30 millions de francs CFA mise à la disposition du maire pour prendre en charge certaines dépenses. Le 7 mars 2017, Khalifa Sall est inculpé pour détournement de deniers publics, d'association de malfaiteurs, de faux et usage de faux. Il est condamné le 30 août 2018 à cinq ans de prison et à la réparation du préjudice de 1,8 milliard de francs CFA (2,74 millions d'euros). Après avoir dirigé la ville de Dakar à partir de la prison de Reubeuss pendant tout le procès, sa condamnation se traduit par sa révocation du fauteuil de maire par le ministre de la Justice.

9 Khalifa Sall, jouissant d'une grande notoriété nationale et internationale ${ }^{2}$, a-t-il eu le tort d'avoir très tôt affiché ses ambitions politiques? Véritable patron de Dakar, que le pouvoir en place peine à arracher de ses mains, Khalifa Sall se présente alors comme un sérieux adversaire de Macky Sall à la présidence de la République de 2019. De fait, l'audit de l'IGE a constitué une opportunité inespérée pour le mettre face à ses responsabilités devant la loi. Les nombreux recours déposés par son pool d'avocats devant les juridictions nationales, pour contester sa condamnation et sa détention arbitraire, et sauver ses chances de candidature à la présidentielle, ont été vains. Son élection comme député aux législatives de juillet 2017 ne lui a pas non plus permis de bénéficier de la liberté provisoire demandée par ses avocats. Demande que la justice a balayée d'un revers de main en arguant de l'antériorité de sa détention. Toutefois, la levée de son immunité parlementaire, le 25 novembre 2017, semble donner raison à ses avocats qui parlent d'une justice au service de la coalition au pouvoir, Benno Book Yakaar. De plus, les nombreux recours non pas empêché la justice de juger cette affaire avant l'échéance de la présidentielle de 2019. Le tribunal de la CEDEAO a d'ailleurs souligné le non-respect des procédures et des droits civiques de Khalifa Sall.

10 Les procès de Karim Wade et de Khalifa Sall laissent planer, dans l'opinion publique, les partis d'opposition et dans la société civile, le sentiment d'une justice sélective et instrumentalisée, qui ne vise que les adversaires du président de la République. De nombreux cas de mauvaise gestion ou de détournement de deniers publics figurent pourtant dans les derniers rapports de l'Office Nationale de Lutte contre la Fraude et la Corruption (OFNAC) et de l'IGE, sans qu'aucune suite judiciaire ne soit donnée ${ }^{3}$. La démission d'un magistrat, celle du juge Dème, afin de fustiger l'instrumentalisation de 
la justice, semble corroborer cet état de fait. Le choix inattendu de gracier Karim Wade, plus populaire en étant à Reubeuss (la prison de Dakar où il est détenu) qu'en liberté, sans recouvrer l'amende qui lui est infligée, a montré que les calculs politiques l'emportent sur la reddition des comptes. Au même moment, la radiation de l'administration d'Ousmane Sonko, leader d'un parti d'opposition, va le propulser dans le champ politique.

\section{Le phénomène Sonko}

11 Fonctionnaire de l'État, Sonko fonde le parti PASTEF (Patriotes du Sénégal pour le travail, l'éthique et la fraternité) en 2014 et développe un discours de contestation et de dénonciation de la mauvaise gouvernance de l'État par une classe politique au service de ses propres intérêts. Convaincu que l'État du Sénégal a été lésé dans les contrats pétroliers et gaziers, il n'a cessé de les dénoncer au point d'y consacrer un livre en 2018, "Pétrole et gaz au Sénégal : chronique d'une spoliation ». L'histoire semble lui donner raison depuis le reportage de la BBC en juin 2019 sur l'affaire Pétro-Tim en vogue dans l'actualité de la presse nationale et internationale. Il s'agit de pots-de-vin qu'aurait reçus Alioune Sall, frère de l'actuel chef de l'État, et l'homme d'affaires Frank Timis, dans le cadre de ces contrats. Le discours de Sonko met à nu les errances dans le fonctionnement de l'État aux plans budgétaire et fiscal. Cela lui a valu la radiation du corps des inspecteurs des impôts de la fonction publique en 2016, pour «manquement au devoir de réserve ». Cette radiation le propulse au-devant de la scène politicomédiatique grâce à un discours mobilisateur portant sur le renouveau de l'action politique adossée à d'autres valeurs comme le « don de soi », son slogan, et l'intérêt de la patrie.

Devenu un phénomène dans le champ politique sénégalais depuis 2016, Sonko est élu député aux législatives de 2017 et se présente de plus en plus comme la seule voie audible dans l'opposition depuis l'emprisonnement de Khalifa Sall. Il le doit surtout à sa virulence et à ses sorties fracassantes à l'égard du pouvoir en place. Il a ravi la vedette à l'ancien Premier ministre, Idrissa Seck, qui ne s'active qu'à l'approche des échéances électorales et au Parti de l'Unité et du Rassemblement (PUR) d'Issa Sall, qui a connu une percée fulgurante aux législatives de 2017. Il a par ailleurs passé l'épreuve du parrainage sans difficulté.

13 La jeunesse et la diaspora sénégalaises voient en Sonko la seule figure de l'opposition en mesure de rompre avec les pratiques d'une classe politique qui, sans cesse, se renie par le phénomène de la «transhumance ${ }^{4}$ » et par le montage de coalitions qui rendent floues les frontières entre socialisme et libéralisme. Il est le candidat le plus suivi dans les réseaux sociaux. La diaspora a apporté une contribution significative dans la mobilisation de sa caution de 30 millions de francs CFA (45 731 euros) à la présidentielle de 2019.

Les campagnes de dénigrement ou de déstabilisation, lancées à son encontre par le pouvoir en place et certains patrons de la presse proches, qui l'accusent de salafisme et d'avoir bénéficié de financements de la part d'un groupe pétrolier britannique, témoignent de son aura auprès de l'électorat et de la crainte qu'il suscite au sommet de l'État. Natif de Thiès, sa jeunesse et son origine casamançaise (la Casamance étant devenue une terre de rébellions indépendantistes depuis 1982) suscitent en revanche la 
méfiance de certains électeurs qui doutent de ses capacités à conduire les destinées du pays à partir de 2019.

\section{L'organisation chaotique des législatives de 2017}

15 C'est dans ce contexte bouillonnant que le pouvoir en place a initié en 2016 une refonte et un renouvellement de la carte d'identité biométrique CEDEAO, qui désormais fait office de carte d'électeur. Ce qui a rendu l'organisation des dernières législatives chaotiques.

Le processus de renouvellement de la carte d'identité nationale, débuté peu de temps avant la tenue des législatives de juillet 2017, est responsable de dysfonctionnements ayant entaché cette élection. L'opposition s'est élevée contre ce calendrier en s'appuyant sur des dispositions de la CEDEAO à propos du changement des règles du jeu des élections dans un délai donné. Les dysfonctionnements portent principalement sur le retrait des cartes, le nombre important de non votants et le décalage entre la production et la distribution de cartes. Malgré les nombreuses annonces du ministre de l'Intérieur, réitérant que tous les électeurs allaient recevoir leur carte à temps, il manquait avant les élections 119000 cartes (CENA, 2019). L'absence de traçabilité et de transparence dans la production et la distribution en est la principale cause (MOE-UE, 2019). Ces dysfonctionnements alimentent l'inquiétude récurrente des partis d'opposition sur la fiabilité du fichier des électeurs, la transparence du processus électoral et l'organisation impartiale des élections. C'est dans ce contexte de crises successives que la loi 2018-14 du 11 mai 2018 a institué le principe du parrainage.

\section{Un parrainage qui reconfigure le champ politique}

17 L'introduction du parrainage citoyen intégral reconfigure le champ politique en réduisant le nombre de candidats. À cela s'ajoute la défection des deux principales formations politiques sénégalaises.

\section{Un parrainage responsable de la réduction du nombre de candidats}

Si le besoin de rationaliser l'éventail des partis politiques, qui dépassent 300 en 2018 , ainsi que le nombre de candidatures aux différentes élections est admis au Sénégal, les modalités pour y parvenir cristallisent les divergences entre le pouvoir en place et l'opposition. Le déroulement des élections législatives de juillet 2017, au cours desquelles pas moins de 47 listes se disputaient 150 postes de députés, justifie cette réforme que le pouvoir en place présente comme une avancée dans l'assainissement de la démocratie sénégalaise.

Introduit au Sénégal en 1963, le parrainage avait un caractère obligatoire. Chaque candidat devait être cautionné par un parti politique ou par dix députés. Ce nombre passa ensuite à cinq, avant que cette mesure ne soit enlevée du code électoral. Toutefois, dans la loi 97-16 du 08 septembre 1997, le parrainage devint ciblé et limité aux candidats indépendants qui doivent être soutenus par au moins 10000 signatures d'électeurs domiciliés dans six régions, à raison de 500 au moins par entité. Le parrainage exprime ici une volonté d'élargir l'offre politique à des candidats n'appartenant pas aux partis traditionnels. En avril 2018, il retrouve son principe 
intégral dans les articles 29 de la constitution et 57 du code électoral. Ainsi, chaque candidature est soumise au parrainage d'une liste d'électeurs représentant entre 0,8\% et $1 \%$ du fichier électoral général, soit environ 53457 signatures à raison de deux mille au moins dans sept des quatorze régions du pays.

La réduction du nombre pléthorique de candidats, et par ricochet, du coût des élections et du temps du vote, donne aux citoyens les moyens de mieux comprendre le paysage électoral. Alors que pour l'opposition elle déconsidère le processus démocratique en accentuant les contraintes liées aux conditions de dépôt des candidatures aux élections. Outre son aspect non consensuel, le parrainage menace, selon certains acteurs de la société civile, la diversité du paysage en mettant hors-jeu les candidats n'ayant aucune base politique; il viole la constitution et vise aussi à l'élimination d'adversaires potentiels en orchestrant le filtrage des candidats à la présidentielle.

L'objectif de réduction du nombre de candidats est en tous cas atteint: on passe de 27 listes potentielles à 5 candidats pour l'élection présidentielle de 2019. En terme d'effectifs, l'élection se rapproche de celle de 1988, pour laquelle seuls quatre candidats se disputaient le pouvoir. En 1993 et 2000, ils étaient huit, mais déjà quinze en 2007 et quatorze en 2012. Parmi les 27 listes ayant déposé leur dossier de parrainage, seul sept ont été validées entre le 26 et le 28 décembre 2018 : Benno Bokk Yakaar (Macky Sall), Idy 2019 (Idrissa Seck), Taxawu Sénégal (Khalifa Sall), Karim Président (Karim Wade), Pastef (Ousmane Sonko), Madické Président (Madické Niang) et PUR (Issa Sall). Parmi les 19 renvoyées pour complément de dossier, aucune n'a réussi à régulariser sa situation dans le délai imparti de 48 heures. Les principaux motifs de rejets évoqués par le Conseil constitutionnel portent sur un problème de report du numéro de carte d'identité de la CEDEAO sur la liste de parrainage, la présence d'un électeur sur la liste de parrainage de deux candidats, le non-respect du quota par région principalement dû à la non-conformité du lieu de vote du parrain figurant sur le dossier de parrainage avec celui indiqué dans le fichier électoral. En effet, le transfert du lieu de vote d'électeurs sans leur consentement serait la principale cause du nombre élevé de rejets (Livre blanc Idy, 2019). Cette pratique apparaît aux yeux de l'opposition comme une ruse du pouvoir en place pour filtrer les candidats.

La création de nouveaux bureaux de vote auxquels étaient affectés 53362 électeurs a accentué l'ire de l'opposition. Sur un fichier électoral de 6683 043, les 27 candidats ont déposé 1414792 parrainages. Les doublons s'élèvent à 174637 et les rejets irremplaçables pour autres motifs se chiffrent à 658106 . Seule la liste Benno Bokk Yaakaar, qui a sollicité les électeurs en premier, a échappé aux doublons. En effet, la liste d'électeurs du parti qui passe en premier constitue la base d'identification des doublons du second, avec des effets cumulatifs pour les candidats qui suivent. Le risque d'obtention de doublons dépend du rang de passage des candidatures au Conseil constitutionnel, qui respecte l'ordre de dépôt. Il est nul chez le premier et va croissant au fur et à mesure qu'on s'éloigne dans l'ordre de dépôt des listes de parrainage. L'ordre de passage des candidats influe sur les rejets pour doublon. Il s'agit d'un classement aléatoire qui ne repose sur aucun critère de représentativité (MOE-UE, 2019).

23 Avec le parrainage, le rôle de la Commission Electorale Nationale Autonome (CENA) est de moins en moins déterminant dans le processus électoral. « Il convient de relever que la CENA n'est pas intervenue dans le contrôle et la validation des parrainages bien que cette séquence soit partie intégrante du processus électoral. D'aucuns ont déploré cette 
situation en rappelant certaines dispositions, notamment les articles L.5 et L. $6^{5}$, du Code électoral » (CENA, 2019). Le ministère de l'Intérieur gère le fichier général auquel les candidats de l'opposition ont des difficultés à accéder. Pourtant, cet accès des partis politiques est une exigence de l'article $48 \mathrm{du}$ Code électoral. Les vérifications techniques du parrainage sont $\mathrm{du}$ ressort du Conseil constitutionnel. Le contexte tendu dans lequel s'est déroulé le parrainage explique le choix du Conseil constitutionnel d'inviter des personnalités indépendantes en qualité d'observateurs, lors des séances de vérification des listes de candidature. "Force est de constater aujourd'hui une mutation des mœurs politiques à travers la substitution au dialogue fécond de monologues parallèles, faits d'invectives et de calomnies dans les médias et les réseaux sociaux. C'est fort de ce constat que le Conseil constitutionnel, [....] conscient que sa mission de sauvegarde de la volonté de l'électeur et de garantie de la sincérité du scrutin doit s'appuyer sur une démarche empreinte de transparence, a ouvert ses activités à la présence des représentants des candidats et à des personnalités sans affiliation connue à une entité politique. Ce choix se justifie par le souci d'instaurer un climat apaisé, non pas au sein de la société dont la sérénité n'a jamais été prise à défaut, mais entre les différents acteurs politiques... $»^{6}$. La CENA se demande aussi pourquoi elle n'a pas été invitée en qualité d'observatrice dans le processus de validation des candidatures, compte tenu de ses prérogatives dans l'organisation des élections.

La vérification des listes de parrainage présentait un caractère plus administratif que juridique Comment contrôler en une semaine la conformité des signatures apposées sur les listes de parrainage de vingt-sept candidats par le Conseil constitutionnel ? Ce travail n'a pas été réalisé. Les listes de parrainages fournis sur clé USB ont fait l'objet d'un traitement automatisé. Les juges du Conseil constitutionnel ne maîtrisent pas le système informatique qui a été conçu en amont. Ils ne peuvent pas vérifier techniquement la fiabilité de ce système et sa neutralité. D'autant plus que les règles et critères de validation des listes n'étaient pas clairs aux yeux de l'opinion et des candidats de l'opposition. Les observations des sept membres de la société civile invités à participer à la vérification des listes de parrainage confirment cet état de fait. «Les limites du système de parrainage tournent autour de la réglementation de l'ordre de passage des candidats à la candidature, l'absence de référentiel précis sur le logiciel de traitement pour éviter les erreurs matérielles, le manque d'informations sur le logiciel des données utilisées et l'absence d'informations détaillées pour les candidats sur les motifs des rejets et autres doublons ${ }^{7}$. La transparence et la fiabilité du système de parrainage se posent. L'absence d'un consensus politique sur l'introduction du parrainage, à moins d'un an de la présidentielle de 2019, a approfondi la crise de confiance entre le pouvoir et l'opposition (MOE-UE, 2019).

\section{La défection des deux partis classiques, le PS et le PDS}

L'élection présidentielle de 2019 est marquée par un fait majeur, l'absence des deux grandes formations politiques du pays, le PS et le PDS, qui ont dominé la vie politique sénégalaise des années 1970 à 2010. Ces deux formations sont traversées par une crise interne provoquée par l'absence de démocratie et par des guerres de positionnement de leurs leaders. L'ancien président Abdoulaye Wade dirige de manière autoritaire le PDS, en imposant son fils, Karim, comme candidat du parti à la présidentielle de 2019. Tous les leaders du parti qui se sont opposés à ce projet sont exclus ou contraints à la démission. D'aucuns ont créé leur mouvement ou parti: Pape Diop, Habib Sy, 
Madické Niang, Aïda Mbodj, Modou Diagne Fada, Abdoulaye Baldé, tous anciens ministres. D'autres ont «transhumé» du côté de Macky Sall: Souleymane Ndéné Ndiaye (ex-Premier ministre), Serigne Mbacké Ndiaye, Samuel Sarr, comme aussi Modou Diagne Fada après avoir créé son propre parti. Dans l'attente de son supposé leader, le PDS a perdu ses hommes les plus influents.

Le 21 mars 2015, l'investiture précipitée de Karim Wade comme candidat officiel du PDS, avant le verdict de la CREI, visait à donner une coloration politique à sa condamnation. Dans la modification du code électoral de 2018, il est précisé que seul un électeur peut être candidat à la présidentielle. Or, Karim Wade n'étant pas inscrit sur les listes électorales et sa demande d'inscription étant rejetée, le PDS se retrouve sans candidat et de fait écarté.

Si le PDS est écarté par voie légale, en revanche, le PS lui renonce à la conquête du pouvoir. Ousmane Tanor Dieng, secrétaire général inamovible du PS depuis le congrès sans débat de 1996, tient à son alliance avec le pouvoir en place, et cela d'autant plus que, au lendemain de chaque élection depuis 2000, son parti perd du terrain. Or l'ancrage du PS dans la coalition Benno Book Yakaar est désapprouvé par certains leaders, notamment Khalifa Sall et Aïssatou Tall Sall, qui veulent se positionner dans la course à la présidentielle. Grâce au verrouillage de l'appareil du PS, Tanor Dieng a fait adopter le soutien du candidat Macky Sall à la présidentielle de 2019, une première. Khalifa Sall et Aïssatou Tall Sall s'affranchissent alors du PS en créant leur mouvement, «Taxawu Dakar» pour le premier et "Osez l'avenir» pour la seconde. L'espoir d'un renouveau du PS porté par le maire de Dakar, Khalifa Sall, s'éloigne. Gagnés par des divisions internes conduisant à leur éclatement en plusieurs mouvements, l'absence du PS et du PDS à la présidentielle de 2019 est révélateur du caractère paternaliste des formations politiques sénégalaises, qui voit le diktat d'un seul homme l'emporter sur la gouvernance interne.

Les condamnations de Khalifa Sall et de Karim Wade et la défection du PDS et du PS ont fortement affaibli l'opposition sénégalaise qui n'avait d'autre choix que de se rassembler pour la campagne.

\section{La formation précoce des coalitions}

L'une des spécificités de l'élection présidentielle de 2019 réside dans la formation précoce de coalitions, bien avant la campagne électorale, alors qu'elles se constituaient généralement au second tour. Le parrainage en est l'une des principales causes. Les vingt-deux candidats recalés au parrainage n'avaient d'autres choix que de se trouver des alliés pour exister. Ces recalés ont surtout gonflé les trois coalitions de l'opposition dirigées par Idrissa Seck, Ousmane Sonko et Madické Niang. D'autres ont choisi la coalition Benno Bokk Yakaar ou se sont abstenus.

D'une coalition d'opposition anti-Wade à une coalition de gouvernement, la durée de vie de Benno Bokk Yakaar étonne. Comment l'APR d'obédience libérale du président Macky Sall peut-elle, sans heurts, cohabiter avec des acteurs centraux de la scène socialiste sénégalaise ? Il s'agit en l'occurrence d'Ousmane Tanor Dieng, mais aussi de Moustapha Niasse, ancien proche de Senghor et fondateur de l'Alliance des forces de progrès (AFP), président actuel de l'assemblée nationale. Le discours de rupture du président Sall, basé sur l'émergence, peut-il s'accommoder du compagnonnage de caciques historiques? C'est l'un des reproches formulés par les Sénégalais à l'encontre 
de Macky Sall. Le parti Rewmi d'Idrissa Seck, d'obédience libérale, a quitté cette coalition qui s'est cependant renforcée avec l'arrivée d'anciens ministres d'Abdoulaye Wade, comme Abdoulaye Baldé, l'édile de Ziguinchor et fondateur de l'Union Centriste du Sénégal, Modou Diagne Fada, Président du mouvement Ldr/Yessal, Cheikh Tidiane Gadio, leader du Mouvement Panafricain et Citoyen Luy Jot Jotna (Il est temps). Mieux, il a reçu le soutien le plus inattendu, celui d'Aïssatou Tall Sall, frondeuse du PS, maire de Podor et présidente du mouvement «Osez l'Avenir ». Après avoir longuement critiqué le choix de Tanor Dieng d'ancrer le PS dans Benno Bokk Yakaar, elle s'est ralliée à Macky Sall en raison, dit-elle, « de la pression maraboutique » du Fouta, sa région natale. Son ralliement n'est pas sans lien avec le parrainage qui a écarté toutes les candidates. Macky Sall a réussi à rallier à sa cause les principaux leaders de la famille libérale au détriment d'Idrissa Seck, l'ex Premier ministre de Wade de 2002-2004.

Parmi les quatre candidats de l'opposition, seul le PUR s'est présenté à la présidentielle sans l'appui d'une coalition. Présidé par Moustapha Sy, guide religieux et leader morale des Moustarchidines, mouvance de la Tidjanya de Tivaouane, le PUR est considéré comme un parti religieux. Il s'en défend en présentant un universitaire, El Hadji Sall, candidat non connoté religieusement, même si les Moustarchidines ont été très actifs dans le cadre de son parrainage et de sa campagne. Les trois autres candidats, Idrissa Seck, Ousmane Sonko et Madické Niang, ont formé des coalitions avec les principaux candidats recalés lors du parrainage (voir Tableau 1). Anticipant le rejet de la candidature de Karim Wade, Madické Niang, avocat et ancien ministre des Affaires étrangères du «Pape du Sopi » (Abdoulaye Wade), se présente comme le candidat par défaut du PDS à la présidentielle.

Tableau 1 - Les coalitions de l'opposition à la présidentielle de 2019

\begin{tabular}{|l|l|l|}
\hline Coalition & Parti du candidat & $\begin{array}{l}\text { Principaux partis ou leader de mouvements de la } \\
\text { coalition }\end{array}$ \\
\hline Idy Président & Rewmi & $\begin{array}{l}\text { Manko Taxawu Sénégal, Khalifa Sall } \\
\text { Bokk Gis Gis, Pape Diop, ancien Président de l'Assemblée } \\
\text { nationale et du Sénat } \\
\text { Cheikh Hadjibou Soumaré, ancien Premier ministre } \\
\text { sous Abdoulaye Wade } \\
\text { Malick Gackou, Grand Parti, l'ex numéro deux (n²) de } \\
\text { l'AFP } \\
\text { Mouvement Agir, Thierno Bocoum } \\
\text { AJ-PADS (And Jëf/Parti africain pour la démocratie et le } \\
\text { socialisme), Mamadou Diop Decroix } \\
\text { Bougane Guèye Dany, PDG du groupe D-média et } \\
\text { président du mouvement Gueum sa bopp }\end{array}$ \\
\hline
\end{tabular}




\begin{tabular}{|c|c|c|}
\hline $\begin{array}{l}\text { Sonko } \\
\text { Président }\end{array}$ & PASTEF & $\begin{array}{l}\text { Pierre Goudiaby Atepa, architecte } \\
\text { «FIPPU », Boubacar Camara } \\
\text { «Yonou askanwi », Madièye Mbodj } \\
\text { Synergies, Mamadou Goudiaby } \\
\text { Téranga Sénégal, Abdoulaye Niane } \\
\text { Union des forces africaines, Bakary Badji } \\
\text { «Juki Diarignou », Daouda Diop } \\
\text { Rassemblement pour un Sénégal nouveau, Abbas Lat } \\
\text { Dior Diop } \\
\text { « Faxas », Gaoussou Koma } \\
\text { «Def Lila War », Yassine Fall }\end{array}$ \\
\hline Madické Niang & $\begin{array}{l}\text { "Jamm Ak Xeweul " } \\
\text { (Paix et Prospérité) }\end{array}$ & $\begin{array}{l}\text { Mouvement Espoir et Modernité / «Yaakaaru Réew mi », } \\
\text { Habib Sy, ancien ministre d'État de Wade } \\
\text { Réconciliation Nationale pour l'Unité Africaine, Ndéla } \\
\text { Madio Diouf } \\
\text { Synergie pour le Progrès et la Démocratie, Matabara } \\
\text { Diop } \\
\text { Union des Groupes Patriotiques/Touba (l'UGP/Touba), } \\
\text { Serigne Modou Bousso Dieng } \\
\text { Tendance "Andou Nawlé », Ibra Diouf Niokhobaye }\end{array}$ \\
\hline
\end{tabular}

\section{Élection : déroulement et résultats définitifs}

Dans un contexte social préélectoral tendu, la campagne de 2019 présentait un risque de violence accrue entre les partisans de la coalition Benno Bokk Yakaar et ceux de l'opposition. Ce qui n'a pas empêché les Sénégalais de voter massivement.

\section{La campagne}

Les candidats ont rivalisé d'ingéniosité en exploitant tous les supports de communication afin de toucher l'électorat : porte-à-porte, médias classiques, réseaux sociaux et affichages à chaque coin de rue, sur les murs, les façades des maisons, les arbres. Les thèmes de campagne mettent l'accent sur les déséquilibres territoriaux, l'accès aux services sociaux de base, le chômage des jeunes, la lutte contre la pauvreté, la gouvernance vertueuse, la solidarité, la restauration des valeurs, la souveraineté monétaire du pays, entre autres. Les vidéos et les messages défilent sur Facebook, WhatsApp et Twitter pour rendre plus vivante la campagne que les utilisateurs suivent en direct dans les différentes régions du pays. Les méthodes de propagandes utilisées par certains candidats avant et durant la campagne électorale ont en effet vite été interprétées comme des appels du pied en direction de leur communauté ou de leur territoire d'appartenance (ou d'origine), donnant ainsi au débat sur le vote identitaire une ampleur particulière. Ceci a été la conséquence du slogan de Macky Sall, «netto ko 
bandoum ", que certains ont perçu comme étant un appel à un vote communautaire ou ethnique. La campagne de 2019 a également été marquée par le séjour de l'ancien président Abdoulaye Wade à Dakar, des scènes de violence, mais surtout par l'humour de Madické Niang, le candidat du Parti de la paix et de la prospérité, qui a décrispé l'atmosphère politique.

En dépit du nombre réduit de candidats, le mépris des autorités du pays pour les mesures allant dans le sens d'une sécurisation de la campagne montre une fois de plus le peu d'intérêt accordé à cette question. Chaque candidat, y compris le président sortant, a recouru au recrutement de nervis, mobilisés sous la forme de milices privées qui ont donné une allure militarisée aux convois de campagne. Quel paradoxe pour des candidats qui se disent aller à la rencontre du simple citoyen avec de tels dispositifs gangstérisés. Les scènes de violences ont opposé des convois de la coalition du président sortant et ceux de l'opposition. Les affrontements à Tambacounda entre un convoi de la coalition Benno Bokk Yakaar et celui du PUR ont choqué l'opinion publique nationale compte tenu de l'ampleur des dégâts (véhicules brulés, saccages), de l'absence des forces de l'ordre et des pertes en vies humaines (deux morts). Chaque camp rejette la responsabilité sur celui d'en face. Le PUR y voit une orchestration du pouvoir en place bâtie sur une double stratégie : la diabolisation et l'arrêt de leur avancée vers le Fouta. Tambacounda a été une opportunité pour décrédibiliser le PUR devant l'opinion nationale, en établissant le lien entre la violence des élections de 1988 à Dakar, opposant les Moustarchidines aux forces de l'ordre, et celle de 2019. La télévision nationale a grandement aidé à cette mise en relation. Cette violence a freiné l'avancée du PUR vers le Fouta où la campagne d'Issa Sall pouvait créer la confusion autour de l'électorat peulh, alors partagé entre deux Sall. Face à cette violence électorale médiatisée, les propos humoristes du candidat Madické Niang (son fameux Fii tojna surtout), partagés via les réseaux sociaux, apparaissent comme une bouffée d'oxygène. Finalement, impatients de reprendre le cours normal de leur vie dans une campagne tendue, certains électeurs ont été plus friands du discours de campagne de ce candidat, teinté de brins d'humours, que des propositions programmatiques des autres.

De l'hôtel cinq étoiles Terrou-bi, sis sur la corniche de Dakar, où Abdoulaye Wade, le "pape du Sopi » séjournait, les médias ont scruté les moindres propos de «Gorgui » (le vieux en wolof). Après un discours inaudible de boycott, puis de sabotage de la présidentielle de 2019, Wade a changé de stratégie. La question a été de savoir s'il allait formuler ou non une consigne de vote pour les militants du PDS au profit d'un candidat de l'opposition. Ousmane Sonko, quoique fervent critique du système de gouvernance mis en place par Wade depuis 2000, a dû stopper sa campagne à l'intérieur du pays pour venir discuter avec Abdoulaye Wade. Finalement, ce dernier a adopté une posture neutre favorable à Macky Sall, qui s'est traduite indirectement par le soutien au candidat sortant (qui en est à son dernier mandat). Ce qui pourrait permettre à son fils, Karim, de se préparer pour la présidentielle de 2024. Wade est finalement venu pour négocier la situation de son fils et non pour soutenir l'opposition. Idrissa Seck, le fils renié, a très tôt compris le jeu de son ex-mentor en misant sur les électeurs qui ont participé massivement à cette présidentielle. 


\section{Localisation et participation des électeurs}

36

seulement en 2019) demeurent des déser
l'électorat national (voir illustration 1).

Illustration 1 - Relation entre le poids démographique et le nombre d'inscrits

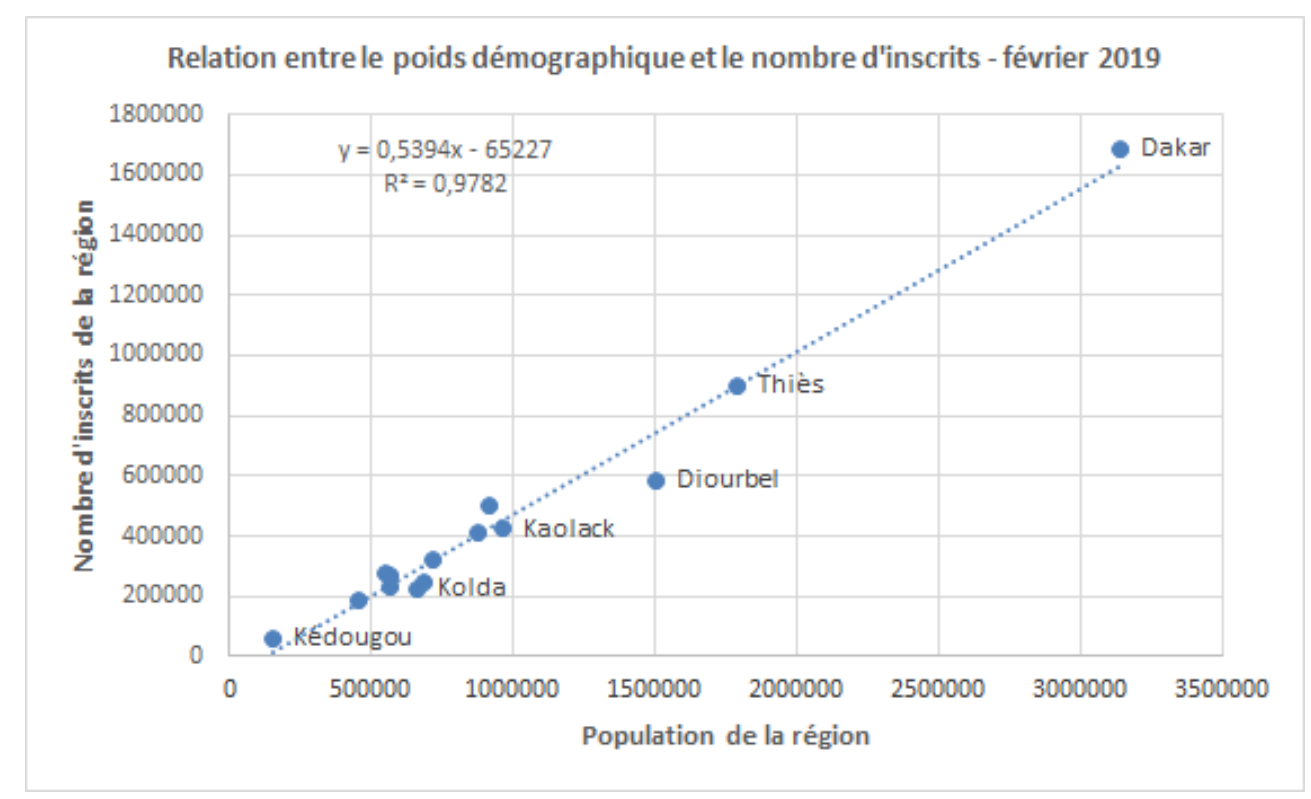

Source : Conseil constitutionnel, février 2019.

Convenons qu'une population électorale importante (nombre d'inscrits sur les listes électorales) n'a de sens que lorsqu'elle contribue à améliorer significativement le taux de participation aux votes. Ce qui n'arrive pas toujours du fait de certaines contraintes susceptibles de gêner le déroulement normal du vote. De ce point de vue, le département de Mbacké, où la participation a été l'une des plus faibles à l'échelle nationale, constitue un exemple patent. En Afrique en général, l'on constate souvent que les conditions d'un vote massif sont rarement réunies dans les circonscriptions électorales peu favorables au régime en place à cause de négligences matérielles ou d'actes de sabotages délibérés (rétention de cartes d'électeurs, nombre limité des bureaux de vote, démarrage tardif du vote), destinés à affaiblir des adversaires gênants. 
Illustration 1 - Élection présidentielle de 2019 : répartition spatiale du vote à l'échelle régionale

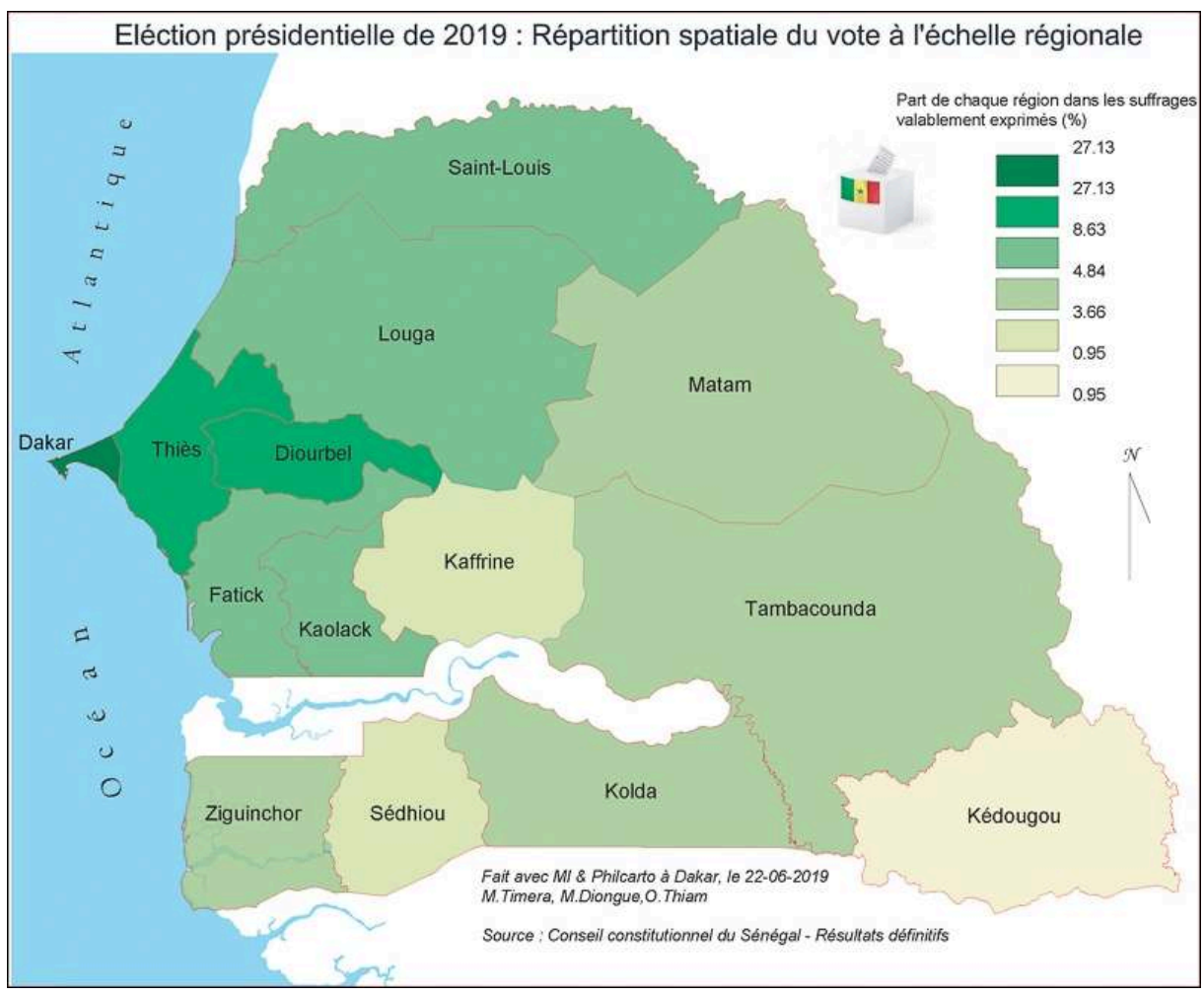

La répartition spatiale des votants à l'échelle départementale montre une configuration un peu différente. Onze départements se détachent nettement du reste et totalisent plus de $56 \%$ des votants. Il s'agit, comme on peut le voir sur la carte qui suit, des départements de Dakar, de Pikine, de Rufisque, de Guédiawaye, de Thiès, de Mbacké, de Mbour, de Tivaouane, de Kaolack, de Podor et de Louga. Il faut noter que, malgré son éloignement géographique, le département de Podor localisé dans la région de SaintLouis reste un fief électoral important, particulièrement courtisé par les candidats aux élections nationales. Les taux les plus importants sont enregistrés dans les départements de Koumpentoum, de Rufisque, de Thiès, de Matam et de Médina Yoro Foulah, avec plus de $70 \%$ de votants. Troisième département du pays en termes de population électorale ( $6 \%$ des inscrits), Mbacké détient l'un des taux de participation les plus faibles, soit $61,7 \%$ contre une moyenne nationale de $66,27 \%$. 
Illustration 2 - Élection présidentielle de 2019 : répartition spatiale du vote à l'échelle départementale

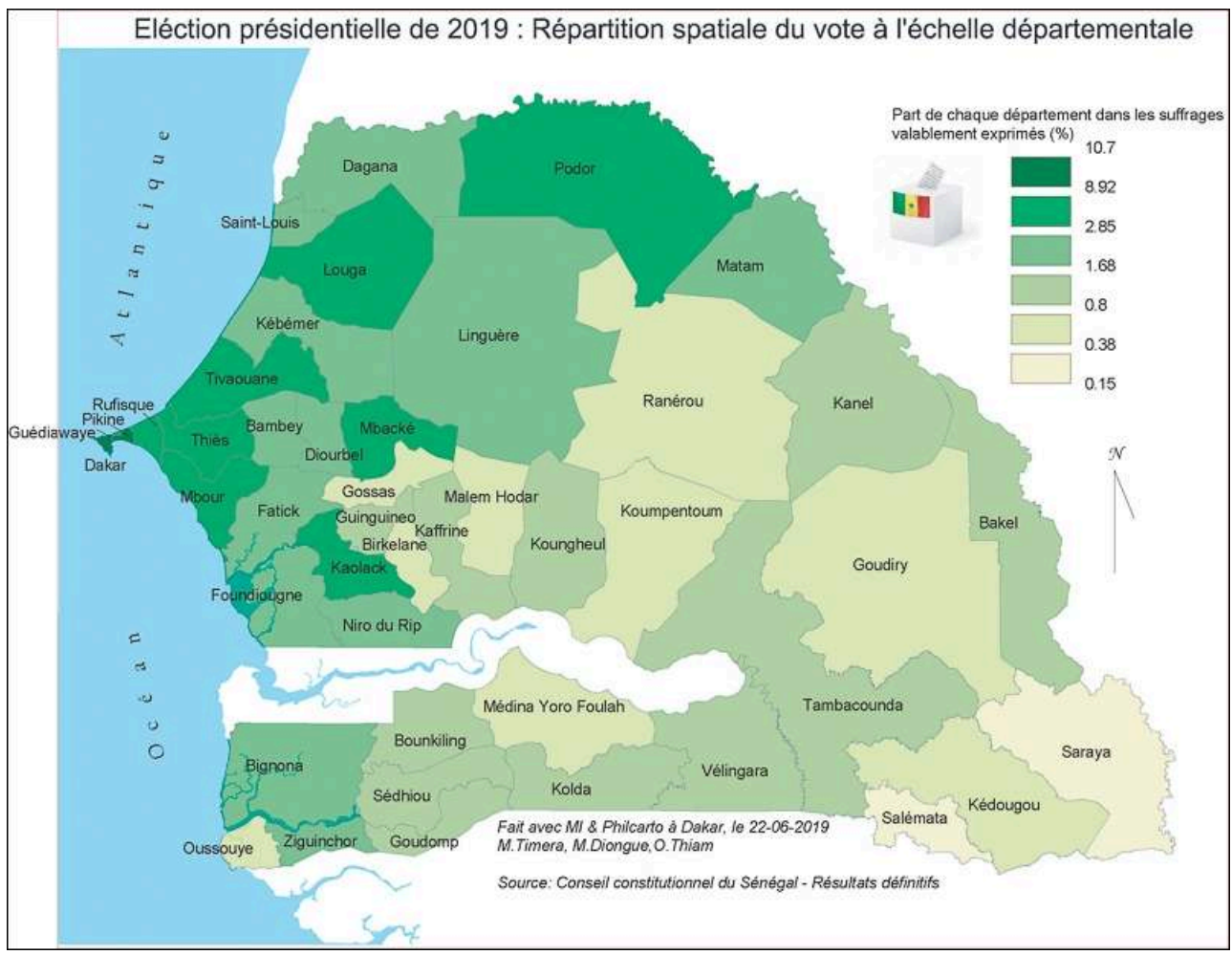

La répartition spatiale du vote au Sénégal, sauf peut-être dans son aspect participation qui devient de plus en plus un phénomène aléatoire, n'échappe pas toujours, comme beaucoup d'autres répartitions spatiales, à la forte concentration de la population près des côtes. La carte électorale demeure caractérisée par une rupture nette entre l'est et l'ouest du pays (où se concentre l'essentiel des électeurs).

\section{L'analyse des résultats des candidats : des soupçons de votes identitaires?}

41 Le candidat Macky Sall a remporté l'élection dès le premier tour avec une majorité nette de 58,26\% des votants. Il est suivi par Idrissa Seck $(20,51 \%)$ et Ousmane Sonko (15,67\%). Les Sénégalais ont opté pour la continuité du Plan Sénégal Émergent (PSE) en 2019, comme lors de la présidentielle de 2007 avec le candidat sortant, Abdoulaye Wade (Magrin, 2007).

Comme on peut le voir dans les graphiques qui suivent, le vote des sénégalais de l'étranger a été assez déterminant pour certains candidats de l'opposition dont il a permis d'améliorer légèrement le score global. Ousmane Sonko est le candidat de l'opposition qui a le plus bénéficié de ce vote. 
Illustration 3a - Résultats globaux de l'élection présidentielle de 2019 - Votes de l'étranger exclus

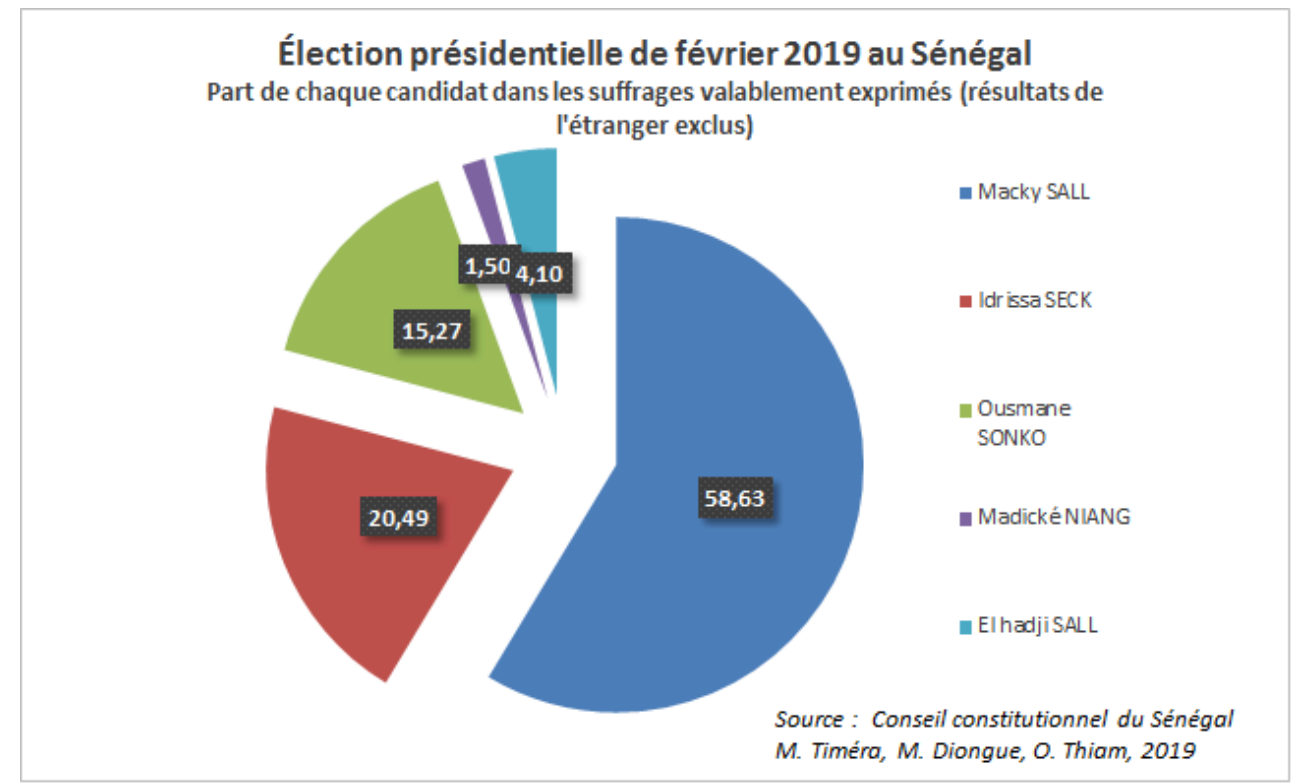

Illustration 3b - Résultats globaux de l'élection présidentielle de 2019 - Votes de l'étranger incluslus

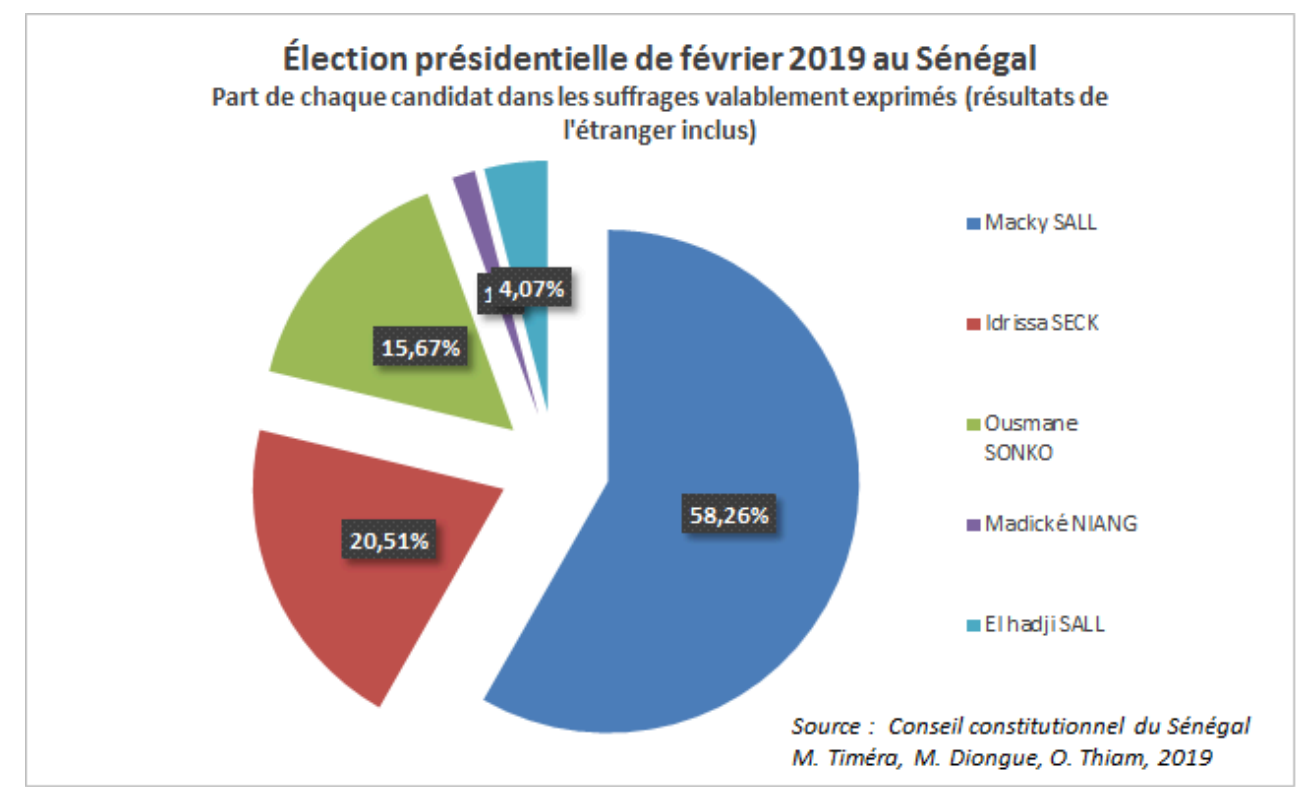

43 La configuration spatiale des résultats finaux de l'élection présidentielle de février 2019, confirme-t-elle l'existence d'un vote identitaire qui a bénéficié à chacun des cinq candidats?

Macky Sall a gagné les principaux départements de la région capitale (Dakar, Pikine et Rufisque), de même que Mbour et Tivaouane. Toutefois, c'est dans les départements des régions majoritairement occupées par ses deux ethnies d'appartenance, Peulh et Sérère, qu'il a obtenu ses scores les plus brillants. Il s'agit des départements de Podor, de Matam, de Fatick et de Kaolack. Ce qui a poussé certains analystes à parler de vote ethnique. Macky Sall est cependant arrivé en tête dans la plupart des départements du pays, avec parfois des scores écrasants. Si la vie politique sénégalaise n'est pas 
organisée autour des clivages ethniques, raciaux ou religieux (Gellar, 2002), le constat d'un vote de soutien ethnique, religieux ou régional semble de plus en plus gagner l'opinion des Sénégalais.

\section{Illustration 4 - Élection présidentielle de 2019 : répartition des votants du candidat Macky Sall}

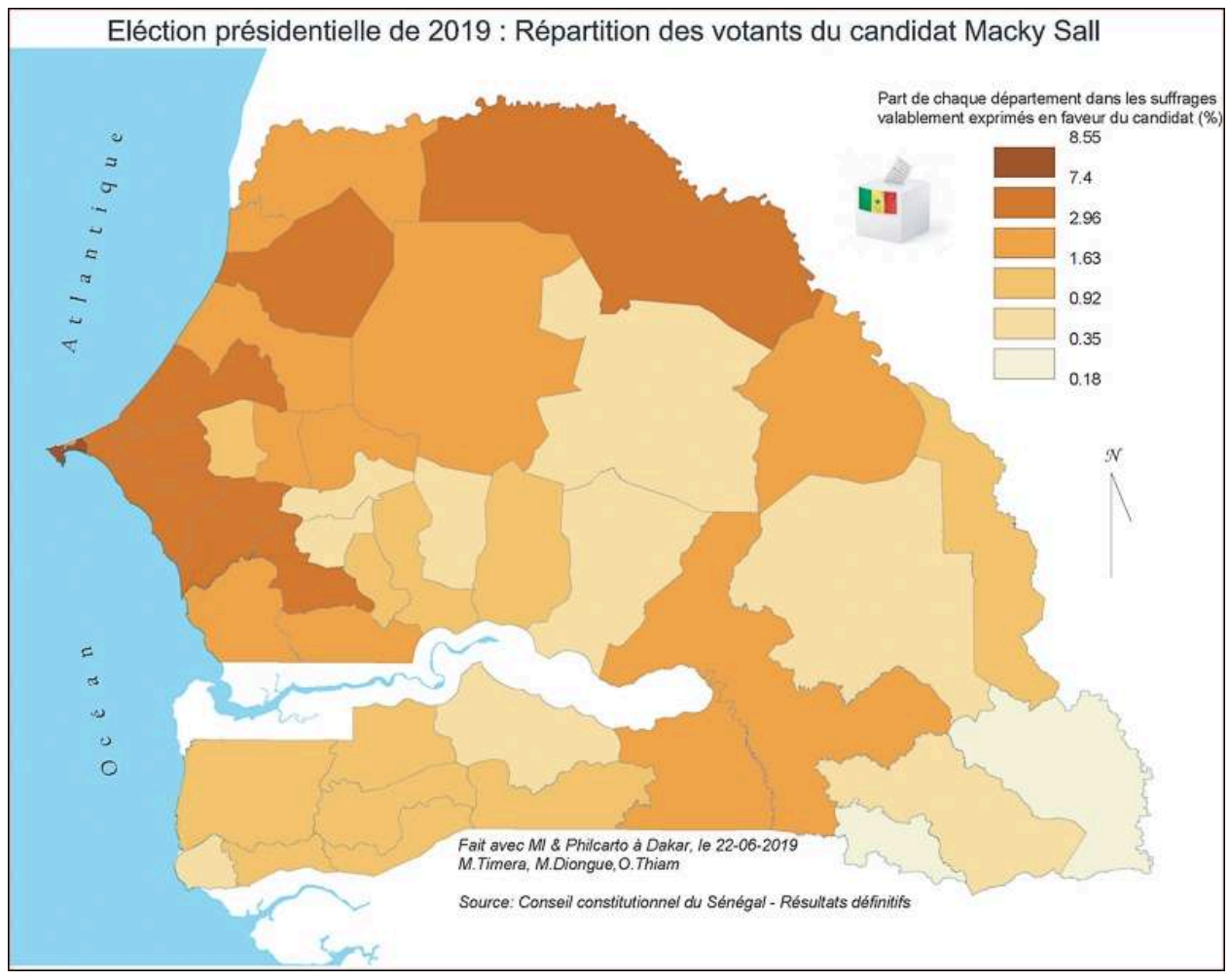

Idrissa Seck a incontestablement bénéficié de la sympathie des Mourides, la communauté religieuse à laquelle il a fait allégeance. Ainsi, c'est au niveau de Mbacké, le département qui abrite le siège de cette communauté, qu'il a réalisé ses résultats les plus brillants. Il s'est nettement distingué dans ce département qui concentre près de $15 \%$ de ses votants au niveau national. Idrissa Seck est également largement arrivé en tête dans le département de Thiès dont il est maire de la commune du même nom, avec $47,15 \%$ des voix contre 39,44 \% pour le candidat Macky Sall. 
Illustration 5 - Élection présidentielle de 2019 : répartition des votants du candidat Idrissa Seck

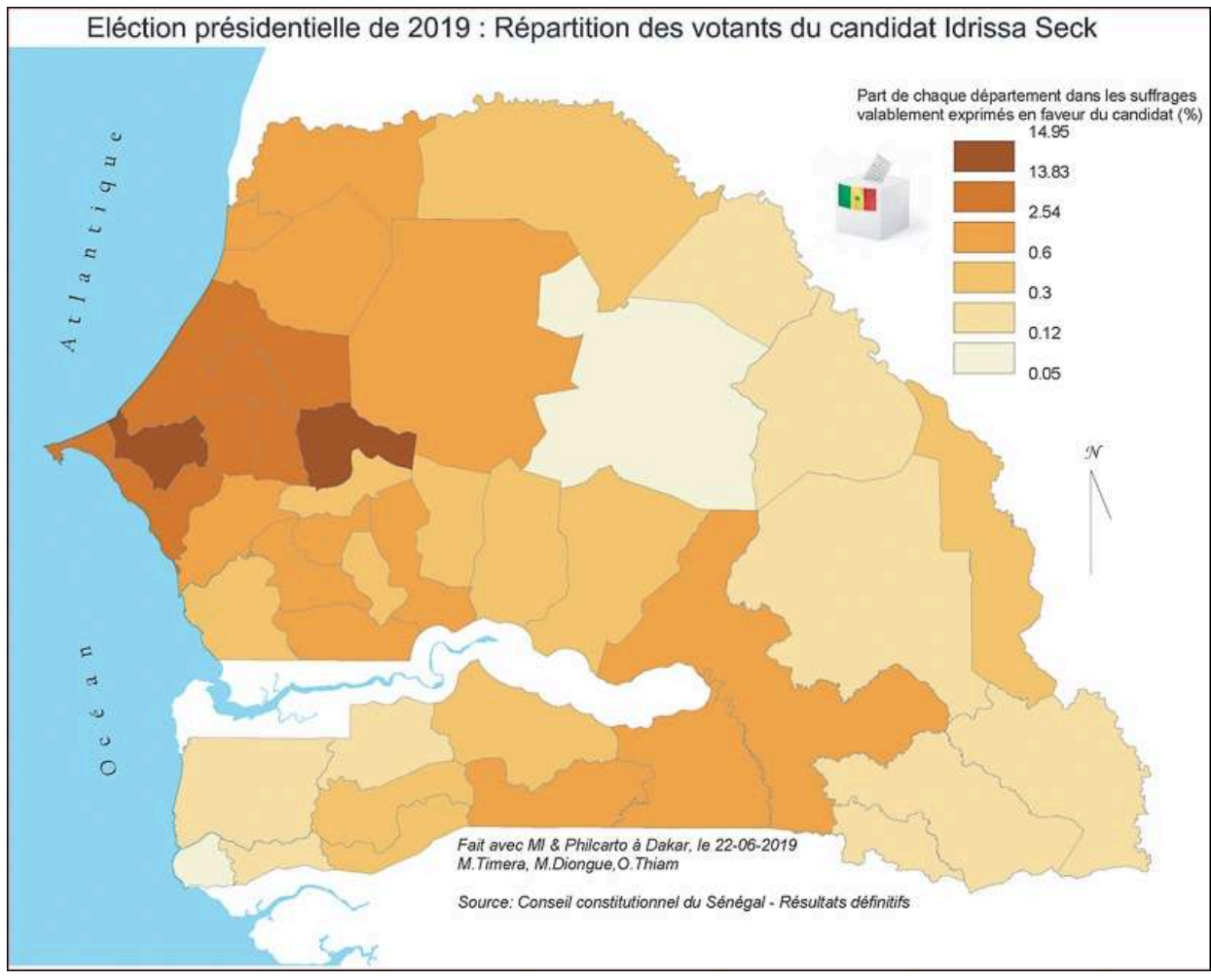

Ousmane Sonko a obtenu ses résultats les plus importants dans les départements des régions de Dakar (où est localisée une bonne partie de ses votants) et de Ziguinchor. Ses scores importants dans cette dernière région dont il est originaire traduisent, selon certains analystes, un véritable élan régionaliste en faveur de ce candidat.

Ses résultats à Dakar, et dans les communes de la banlieue de l'agglomération plus particulièrement, s'expliquent sans doute par son statut de candidat pivot de la contestation populaire à l'encontre du pouvoir en place. Ses diatribes régulières à l'encontre du régime de Macky Sall lui ont permis de constituer un capital de sympathie immense auprès de l'électorat des milieux défavorisés, majoritairement localisé dans les quartiers de ces communes. C'est cette posture particulière qui entretient chez lui l'image d'un candidat antisystème. 
Illustration 6 - Élection présidentielle de 2019 : répartition des votants du candidat Ousmane Sonko

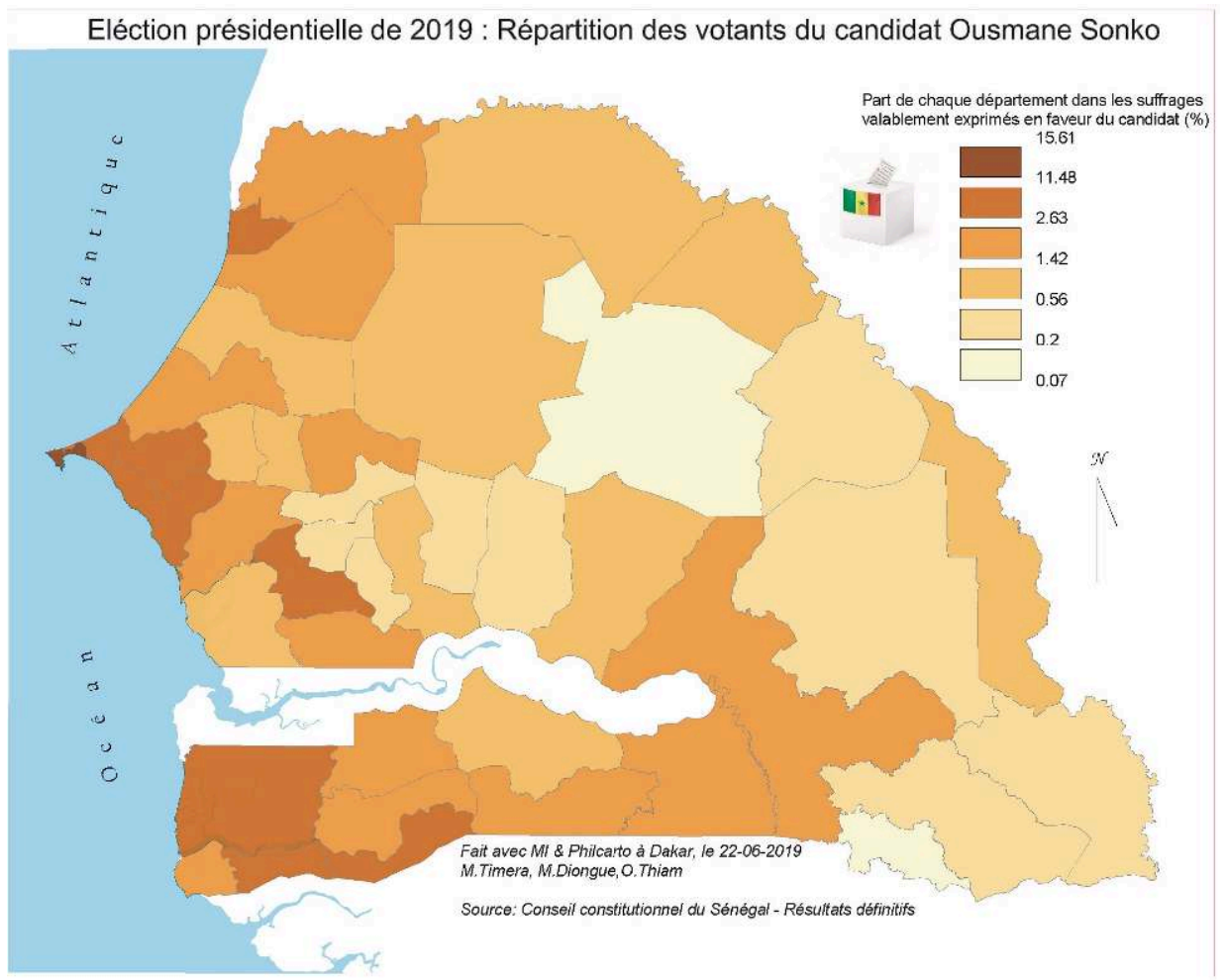

Comme les candidats Idrissa Seck et Madické Niang, Issa Sall a, lui aussi, bénéficié d'un vote religieux. C'est dans les départements où sont majoritairement localisés les Moustarchidines, une communauté religieuse avec laquelle il entretient des liens privilégiés, qu'il a obtenu ses meilleurs résultats. Il s'agit notamment des départements de la région de Dakar et de ceux de Tivaouane, Louga, Kébémer, Saint-Louis et Dagana.

La particularité de l'électorat du PUR, le parti du candidat Issa Sall, est qu'il reste encore majoritairement localisé en milieu urbain - les villes des départements cités cidessus en particulier - où la communauté moustarchidine a pris naissance sous forme de dahiras (associations religieuses), avant de se développer dans le reste du pays. Son électorat rural est encore peu nombreux, ce qui, en partie, explique ses faibles résultats dans les départements de l'intérieur. 
Illustration 7 - Élection présidentielle de 2019 : répartition des votants du candidat Issa Sall

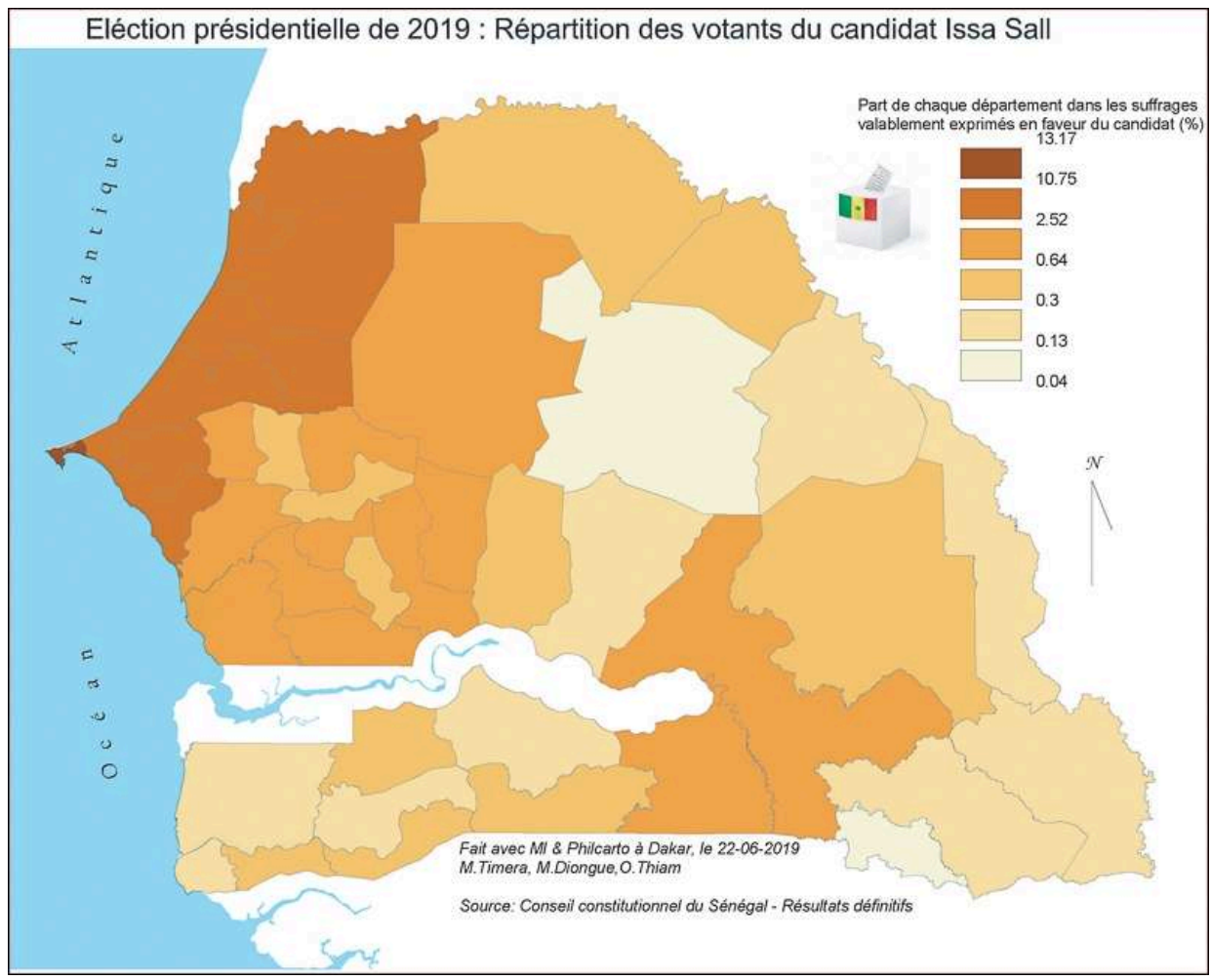

Les votants de Madické Niang sont essentiellement localisés dans les départements de Pikine et de Mbacké où il a réalisé ses meilleurs résultats. Dans ce dernier, le candidat Madické Niang a bénéficié de la forte sympathie des Mourides, communauté à laquelle il a fait allégeance. Cependant, la grosse surprise pour ce candidat vient de Saint-Louis, son département natal qu'il n'a pas gagné et où il a obtenu un très faible résultat comparé à ceux des autres candidats.

Les médiocres résultats de Madické Niang dans beaucoup de départements pourraient s'expliquer par les soupçons de connivence avec le candidat Macky Sall, qui ont pesé sur lui durant toute la campagne et qui ont abouti au fait qu'il n'a pas convaincu beaucoup d'électeurs en dépit de ses déclarations de bonne foi. Beaucoup d'entre eux pensaient en effet que Madické Niang n'était rien d'autre que le candidat déguisé d'Abdoulaye Wade, dont l'imperturbable neutralité a été interprétée par beaucoup comme une manière de soutenir le candidat Macky Sall qui serait disposé à amnistier son fils Karim Wade en cas de victoire.

Illustration 8 - Élection présidentielle de 2019: répartition des votants du candidat Madické Niang 


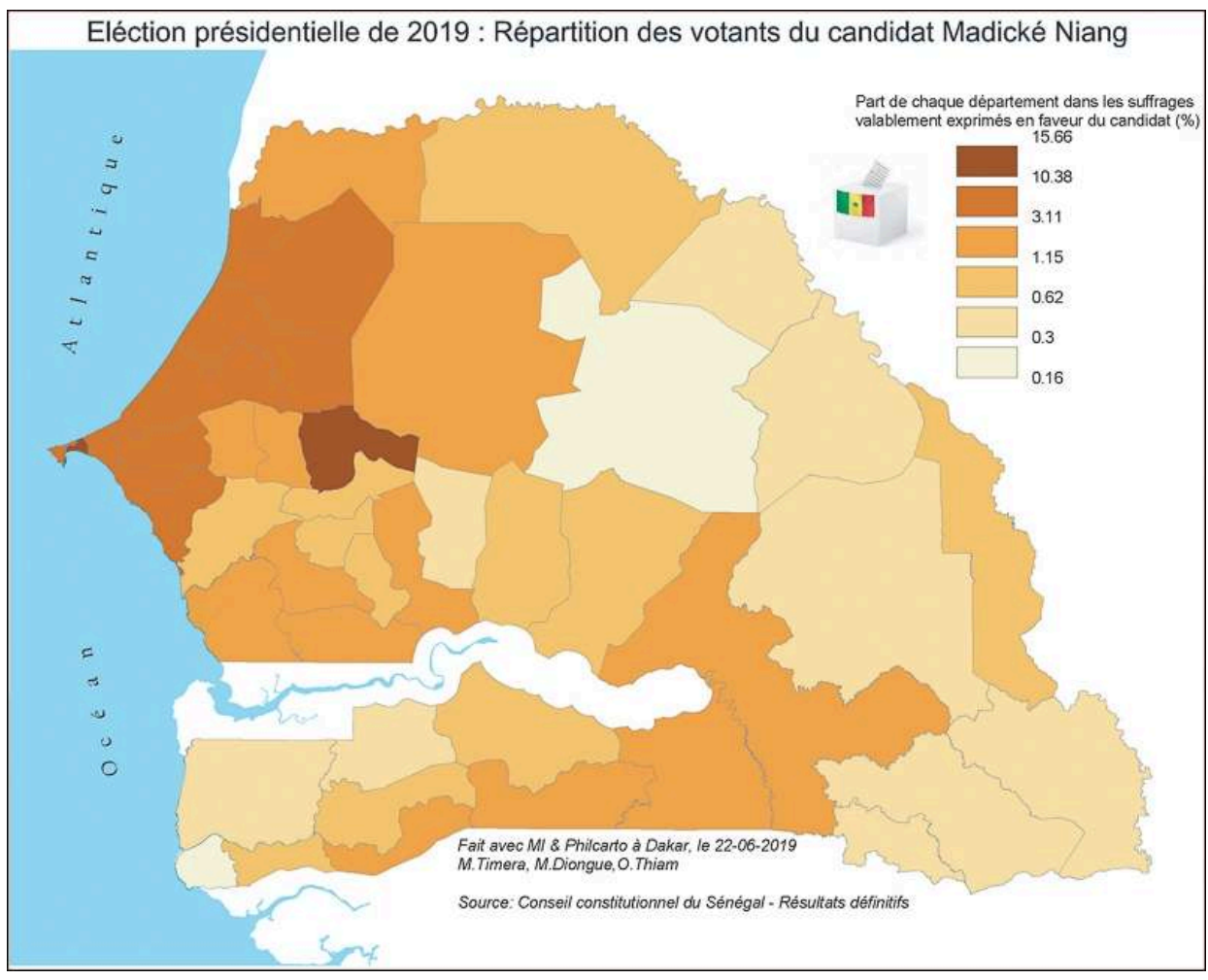

Les Sénégalais de l'extérieur ont en majorité voté pour l'opposition, soit au total $62 \%$. C'est seulement en Afrique de l'Ouest que Macky Sall l'emporte largement avec $58 \%$. Sonko se présente comme le principal candidat de l'opposition à l'étranger. Il est arrivé en deuxième position derrière le président sortant en Europe de l'ouest, du centre et du nord, où les deux candidats totalisent respectivement $41 \%$ et $47 \%$ des suffrages. Sonko devance Macky Sall en Amérique avec un score de $46 \%$ des votes. 


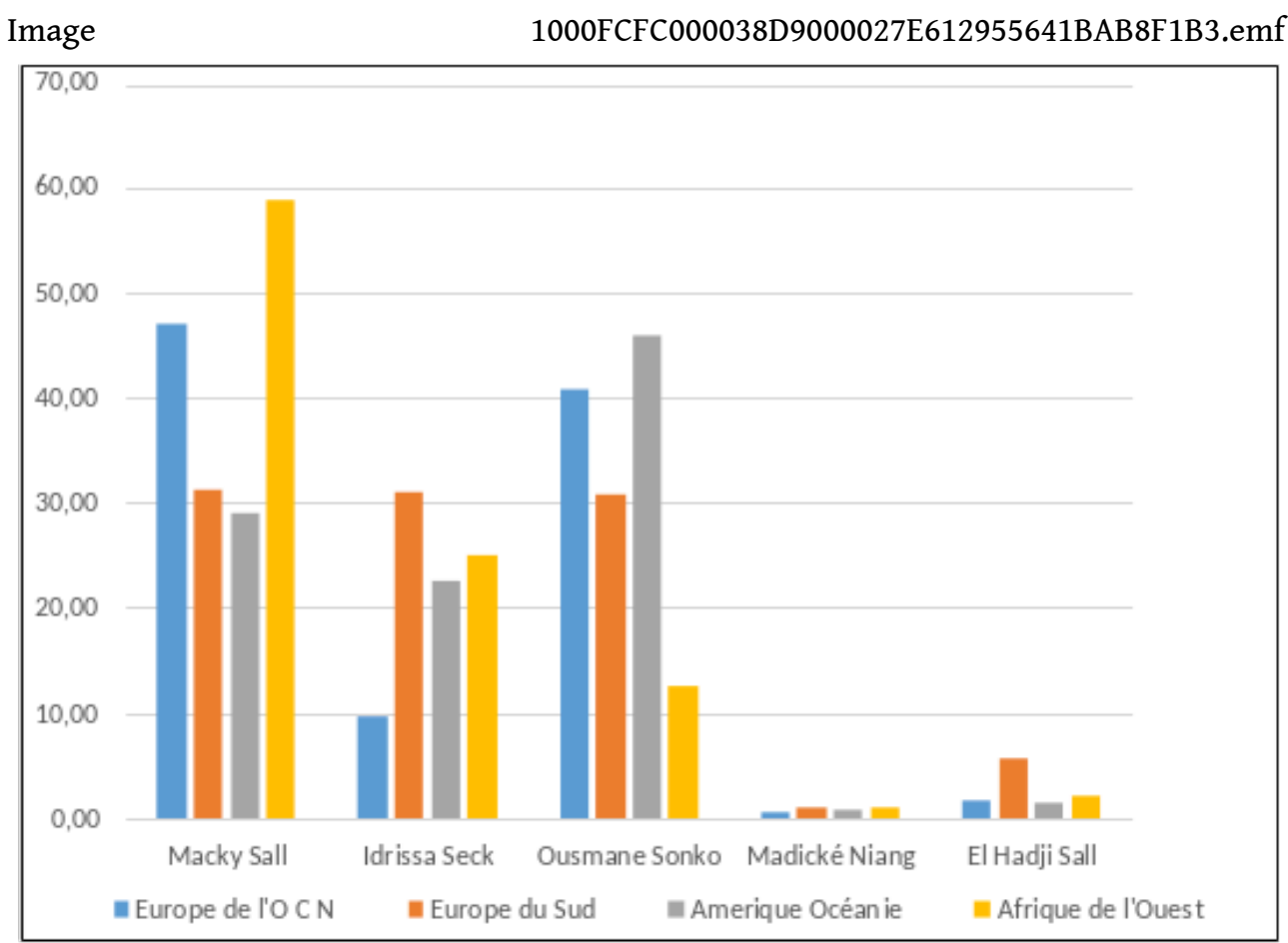

Source : Conseil constitutionnel, résultats globaux, février 2019.

\section{Conclusion}

La tension post-électorale qui est née dans les états-majors des partis politiques, lors de la proclamation des résultats, reste le point d'orgue d'un contexte où frustrations, positionnements, oppositions ou partis pris politiques ont marqué la vie politique du Sénégal depuis 2001. Du côté de l'opposition, les guerres de positionnement internes, qui sont les effets collatéraux soit de l'absence du PDS à la présidentielle de 2019, soit du compagnonnage avec le pouvoir (PS, AFP), ont entretenu tout le long du processus une double tension : à l'intérieur des partis et entre l'opposition et le régime en place. Quant au pouvoir, son emprise sur la justice a été déterminante. Aussi bien dans le fond que par le calendrier, les affaires dites politico-judiciaires ont fini par faire accroitre le sentiment d'une instrumentalisation juridique, y compris au sein de l'appareil judiciaire, dont l'objectif a été d'écarter des adversaires et de baliser la voie à une réélection.

Le parrainage présenté par le pouvoir comme un moyen de rationaliser l'offre pléthorique de partis politiques a été source de désaccords majeurs. Outre la réduction du nombre de candidats, il a permis de redessiner le paysage politique, notamment par l'élimination des candidatures féminines, et par la formation précoce des coalitions qui ne se constituaient généralement qu'au second tour. Le parrainage a eu surtout comme effet d'unir l'opposition qui s'est rassemblée autour du refus du système imposé en formant des coalitions, mais aussi en défiant le pouvoir en place à travers des prises de positions ponctuelles comme l'organisation d'une conférence conjointe, après la fermeture des bureaux de vote et l'annonce des premiers résultats dans la soirée, d'Idrissa Seck et d'Ousmane Sonko. 

pas montré de grandes disparités géographiques entre elle et l'opposition réunie. À l'échelle de la région dakaroise, l'opposition est créditée d'une avance de plus de 25175 voix, ce qui conforte l'idée d'une faible hégémonie territoriale. La capitale sénégalaise et son hinterland immédiat restent en conséquence à conquérir pour le pouvoir. Convaincue d'un équilibre territorial des rapports de force lors du vote, l'opposition l'est plus par l'existence de "territoires conquis ", ce qui est un paradoxe de la configuration territoriale du vote. Le vote régional ou le régionalisme électoral a été, sauf pour la coalition de Madické Niang, la marque de cette élection présidentielle et a davantage fondé pour l'opposition la certitude d'un poids électoral significatif. L'opposition partage toutefois ce sentiment avec le pouvoir qui, par la voie du directeur de la compagnie de transport public Dakar Dem Dick, clamait que le Fouta (région de Matam), compte tenu de son poids dans la réélection du président, méritait plus l'autoroute à péage Ila Touba que cette dernière et ses environs qui ont voté pour l'opposition.

Même si des liens existaient entre la presse et le monde politique, les médias restent perçus comme des sentinelles de la démocratie. Avec 2019, les positions et les partispris sont plus tranchés puisque les patrons de presse (Youssou Ndour, Bougane Guèye Dani) sont devenus des acteurs clés et partie prenante de la vie électorale. Ceux qui n'étaient pas des leaders politiques, comme Madiambal Diagne et Aziz Ndiaye, ont manifesté directement ou indirectement leur préférence pour des coalitions politiques, au point d'installer des remous au sein de leurs rédactions (le journal Le Quotidien, la chaîne $2 s t v)$. Cette posture des médias privés a contribué à exacerber via les émissions de télévision les tensions post-électorales. La couverture médiatique de la présidentielle de 2019 est empreinte d'une forte dose de partialité. Cette attitude des médias est aux antipodes de leur rôle de sentinelle de la démocratie et de sauvegarde du vote des électeurs lors des présidentielles de 2000 ( $1^{\text {ère }}$ alternance) et de 2012 ( $2^{\text {ème }}$ alternance).

L'élection présidentielle de 2019 a été atypique. La gestion du processus électoral a été chaotique. L'interprétation des résultats ou la gestion immédiate du vote a été source de vives tensions entre le pouvoir en place et l'opposition. L'élection présidentielle de 2019 pointe du doigt le paradoxe suivant : elle reste marquée par l'instabilité politique et institutionnelle, alors même que la démocratie sénégalaise est considérée comme apaisée. Le report des élections locales de 2019 pointe également le problème des parrainages.

Le début du second mandat de Macky Sall, chaotique et marqué par la suppression du poste de Premier ministre ainsi que par l'affaire du pétrole et du gaz qui touche son frère, Aliou Sall, l'oblige à lancer un dialogue national. L'inauguration de la grande mosquée mouride de Dakar, Massalikoul djinane, le 29 septembre 2019, a été marquée par les chaleureuses salutations d'Abdoulaye Wade et de Macky Sall, et par la libération de Khalifa Sall, gracié le lendemain. Ce qui ouvre la voie à de potentiels retrouvailles entre l'ex-président Wade et l'actuel, et à un possible retour du fils exilé, Karim Wade. Ceci ne sera pas sans effet sur le jeu politique sénégalais caractérisé par l'imprévisibilité et l'instabilité. 


\section{BIBLIOGRAPHIE}

Agence de presse sénégalaise (APS), 2018. Les "admis" et les "recalés" de la vérification des parrainages font l'actualité. 31/12/2018. http://news.adakar.com/h/104981.html

Ba M., 2017. Sénégal : inculpé pour détournement de deniers publics, Khalifa Sall a été incarcéré. Jeune Afrique, 8/03/2017. https://www.jeuneafrique.com/410466/politique/senegal-inculpedetournement-deniers-publics-khalifa-sall-a-ete-incarcere/

Baldé I., 2019. Sonko face à de nombreux défis. Sud Quotidien [En ligne], 2 mars 2019. https:// www.sudonline.sn/sonko-face-a-de-nombreux-defis_a_43138.html

CENA (Commission Electorale Nationale Autonome). Rapport sur l'élection présidentielle du 24 février 2019. [Dakar], $184 \mathrm{p}$.

Coalition Idy 2019. Rapport public sur l'élection présidentielle du 24 février 2019 : observations saillantes autour d'un hol-dup électoral. [Dakar] , 59 p.

Conseil constitutionnel du Sénégal, 2019, Résultats définitifs de l'élection présidentielle du 24 février 2019.

Coulon C., 1992. La démocratie sénégalaise : bilan d'une expérience. Revue Politique africaine, $\mathrm{n}^{\circ} 45$, p. 1-9.

Dia M., 2019. Le parrainage au Sénégal. Ce couteau à double tranchant. Sud Quotidien [En ligne], 2 janvier 2019. http://www.sudonline.sn/le-parrainage-au-senegal--ce-couteau-a-double tranchant_a_42380.html

Gellar S., 2002. Pluralisme ou jacobinisme : quelle démocratie pour le Sénégal ? In DIOP M. C. (dir.), Le Sénégal contemporain. Karthala, Paris, p. 507-528.

Jeune Afrique - AFP, 2018. Sénégal : l'opposition manifeste pour une présidentielle juste en 2019 [En ligne], 10 février 2018. https://www.jeuneafrique.com/529561/politique/senegal-loppositionmanifeste-pour-une-presidentielle-juste-en-2019/

Magrin G., 2007. Sopi or not sopi ? A propos des élections présidentielles de février 2007 au Sénégal. EchoGéo [En ligne], Sur le Vif, mis en ligne le 20 juin 2007. http:// journals.openedition.org/echogeo/838 - DOI : 10.4000/echogeo.838

Maillard M., 2018. Sénégal : la révision du code électoral votée dans un climat de forte contestation. Le Monde Afrique, 19/04/2019 [En ligne]. https://www.lemonde.fr/afrique/article/ 2018/04/19/senegal-la-loi-sur-le-parrainage-votee-dans-un-climat-de-fortecontestation_5287952_3212.html

Maillard M., 2019. Élection présidentielle 2019 au Sénégal : Macky Sall a rétréci l'opposition. Le Monde Afrique [En ligne], 22 février 2019. https://www.lemonde.fr/afrique/article/2019/02/22/ au-senegal-macky-sall-a-retreci-l-opposition_5426705_3212.html

MOE-UE (Mission d'Observation Electorale Union Européenne), 2019. Sénégal, Rapport final élection présidentielle de février 2019, 77 p. [En ligne]. senegal_moe_ue_2019_rapport_final.pdf

Ndiaye A. S., 2019. Campagne présidentielle 2019 et marketing politique : radiographie des stratégies 2.0 des candidats, Socialnetlink.org [En ligne], 5 mars 2019. https:// www.socialnetlink.org/2019/03/campagne-presidentielle-2019-et-marketing-politiqueradiographie-des-strategies-2-0-des-candidats/ 
P., 2018. Conseil Constitutionnel : Liste des candidats recalés au parrainage. Dakarmidi, 31/12/2018 [En ligne]. https://www.dakarmidi.net/actualite/conseil-constitutionnel-liste-descandidats-recales-au-parrainage/

Rassoul E., 2019. Présidentielle 2019 : une campagne en arc-en-ciel. Au-Senegal.com [En ligne], 18 février 2019. https://www.au-senegal.com/presidentielle-2019-une-campagne-en-arc-en-ciel, 15615.html

Sene D., 2018. Présidentielle 2019 du Sénégal : invité de l'Agence APA, Alioune Tine souligne les «faiblesses » du parrainage. Lactuacho.com [En ligne]. http://www.lactuacho.com/ presidentielle-2019-du-senegal-invite-de-lagence-apa-alioune-tine-souligne-les-faiblesses-duparrainage/

SenePlus Medias - APS, 2019. La violence liée à la campagne électorale en exergue. 12 février 2019. https://www.seneplus.com/media/la-violence-liee-la-campagne-electorale-en-exergue

SineSaloum, 2019. 27 candidats enregistrés : le « parainage » contourné! [En Ligne]. http:// www.sinesaloum.info/27-candidat-enregistres-le-parrainage-contourne.html

Soudan F., 2010. La leçon de Dakar. Dossier Jeune Afrique, 31 mars 2010.

The World News, 2018. Parrainage à mi parcours. Les recalés et les admis [En ligne], 30 décembre 2018. https://theworldnews.net/sn-news/parrainage-a-mi-parcours-les-recales-et-les-admis

\section{NOTES}

1. Ce refus a été confirmé par la cour d'appel de Paris. Le comité des droits de l'homme de l'ONU s'est prononcé contre l'État du Sénégal.

2. Au moment de son arrestation, il était, entre autres, président de l'Alliance mondiale des villes (Cities alliance), secrétaire général de l'Association Internationale des maires francophones.

3. Les centaines d'emplois fictifs du CROUS et le supposé détournement de 29 milliards de Francs CFA dans le cadre du PRODAC défraient la chronique depuis longtemps. Ces deux affaires concernent des ministres de l'actuel gouvernement de Macky Sall.

4. La «transhumance » décrit, dans le champ politique, cette propension de certains leaders de partis à quitter leur formation pour gonfler les rangs du parti au pouvoir après chaque élection présidentielle.

5. «La CENA est obligatoirement présente à tous les niveaux de conception, d'organisation, de prise de décision et d'exécution depuis l'inscription sur les listes électorales jusqu'à la proclamation provisoire des résultats ».

6. Discours du président du Conseil constitutionnel du Sénégal, Pape Oumar Sakho, à l'occasion de la prestation de serment du président de la République du Sénégal, M. Macky Sall, le 02 avril 2019.

7. http://aps.sn/revue-de-presse/article/les-manquements-deceles-dans-le-parrainage-a-la-une 


\section{RÉSUMÉS}

L'article analyse l'élection présidentielle de février 2019 au Sénégal. Il décrit le contexte préélectoral et met l'accent sur le processus et les résultats du scrutin avec en retour un regard sur les implications $\mathrm{du}$ contexte de déroulement. L'analyse combine deux approches méthodologiques: une approche interprétative fondée sur l'observation du contexte et une approche descriptive qui s'appuie sur le recueil et la cartographie des données statistiques publiées par le Conseil constitutionnel du Sénégal. Les résultats de l'étude montrent que l'élection présidentielle et son contexte ont été marqués par une crise politico-judiciaire qui a favorisé une reconfiguration du paysage politique avec la disparition de la compétition de partis historiques et l'émergence d'une nouvelle figure politique, chantre de l'« antisystème ». Même si le candidat du parti au pouvoir a été reconduit, les résultats du vote n'ont pas montré de grandes disparités géographiques entre le pouvoir et l'opposition réunie. Convaincue d'un équilibre territorial des rapports de force au moment du vote, l'opposition l'est encore plus par l'existence de «territoires conquis », ce qui présageait une crise postélectorale. De plus, le vote régional ou le régionalisme électoral tout comme la prise de position partisane des médias ont été des aspects essentiels de cette élection.

The article analyzes the presidential election of February 2019 in Senegal. It describes the preelection context and focuses on the process and results of the polls with a look back at the implications of the unfolding context. The analysis combines two methodological approaches: an interpretative approach based on context observation and a descriptive approach based on the collection and mapping of statistical data published by the Constitutional Council of Senegal. The results of the study show that the presidential election and its context were marked by a politicojudicial crisis that favored a reconfiguration of the political landscape with the disappearance of the competition of historical parties and the emergence of a new political figure which is the forerunner of the "antisystem". Although the ruling party's candidate was renewed, the results of the vote did not show large geographical disparities between the power and the opposition. Convinced of a territorial balance of power in the vote, the opposition is more by the existence of "conquered territories", which foreshadowed a post-election crisis. In addition, the regional vote or electoral regionalism as well as the partisan position of the media were essential aspects of this election.

\section{INDEX}

Keywords : Senegal, presidential election, sponsorship, post-electoral tension, political reconfiguration, media, opposition, politico-judicial crisis

Mots-clés : Sénégal, élection présidentielle, parrainage, tension postélectorale, reconfiguration politique, média, opposition, crise politico-judiciaire

\section{AUTEURS}

\section{MAMADOU BOUNA TIMERA}

Mamadou Bouna Timera, mamadou.timera@ucad.edu.sn, est maître de conférences à l'Université Cheikh Anta Diop de Dakar (Sénégal). Il a récemment publié :

- Timera M. B. et al., 2018. Champ épistémique des travaux des géographes Paul Pélissier et 
Cheikh Ba : entre complémentarités, recoupements et débordements disciplinaire. Cahiers de géographie du Québec, vol. 62, n 175, p. 169-189.

- Timera M. B. et al., 2016. Islam et production des espaces urbains au Sénégal : les mosquées dans la périphérie de Dakar (Keur Massar extension). Germivoire, Revue scientifique de la littérature, des langues et sciences sociales, $n^{\circ} 4$, p. 226-244

- Timera M. B., 2012. Le Japon, objet d'étude et d'enseignement au Sénégal. Cybergeo : European Journal of Geography [En ligne], document 609. http://journals.openedition.org/cybergeo/25372 - DOI :10.4000/cybergeo.25372

\section{MOMAR DIONGUE}

Momar Diongue, momar.diongue@ucad.edu.sn, est maître assistant en Géographie urbaine, à l'Université Cheikh Anta Diop de Dakar (Sénégal). Il a récemment publié :

- Diongue M., Diouf S., 2018. Urbanisation des périphéries urbaines et risques d'inondation à Dakar (Sénégal). Le cas de la commune de Yeumbeul Nord. In Cisse O. (dir.), Les inondations à Dakar : gestion des risques et adaptations locales. Paris, Karthala, p. 37-76.

- Timera M. B., Diongue M. et al., 2018, Champ épistémique des travaux des géographes Paul Pélissier et Cheikh Ba : entre complémentarités, recoupements et débordements disciplinaire. Cahiers de géographie du Québec, vol. 62, nº 175, p. 169-189.

- Mboup G., Diongue M., Ndiaye S., 2017. Dakar : Smart City Foundation-Driver of Smart Cities. In Vinod Kumar L. M. (éd.), Smart Economy in Smart Cities : International Collaborative Research. Springer Singapore, p. 841-869.

\section{OUSMANE THIAM}

Ousmane Thiam, ousmane.thiamsn@gmail.com, est docteur en géographie et expert en aménagement urbain et développement territorial à l'Agence nationale de l'aménagement du territoire (ANAT)-Sénégal. Il a notamment contribué aux publications :

- ONU-Habitat/UNEP, 2010. L'état des villes africaines. Gouvernance, inégalités et marchés fonciers urbains. 279 p. [En ligne] http://www.zaragoza.es/contenidos/medioambiente/onu/535-freed2010.pdf

- Denis E. et al., 2008. Dynamiques de l'urbanisation, 1950-2020: approche géostatistique. Afrique de l'Ouest. Agence Française de Développement (AFD), 124 p. + atlas. https://hal.archivesouvertes.fr/hal-00357271/file/Africapolis_1_Rapport.pdf 